\title{
ENTREVISTA CON PROF. Dr. CLAUDIO RAMA
}

\author{
INTERVIEW WITH Dr. CLAUDIO RAMA
}

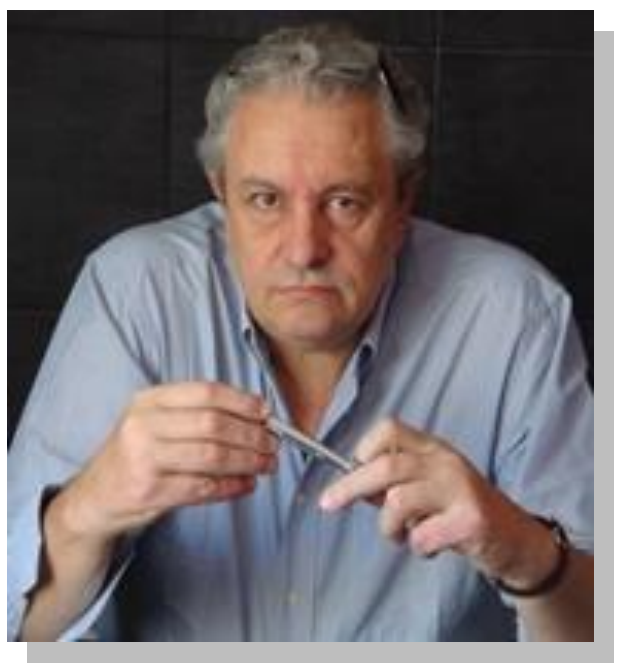

\author{
"Más allá de las horas mínimas de clase, \\ existen enormes niveles de diferencia de los \\ programas y de los sistemas de educación \\ superior en América Latina y específicamente \\ en el MERCOSUR que imposibilitan un \\ reconocimiento automático de las \\ titulaciones" (Claudio Rama).
}

\section{Claudio Rama ${ }^{1}$ \\ Maria de Lourdes Pinto de Almeida ${ }^{2}$ José Camilo dos Santos Filho ${ }^{3}$}

\section{Presentación}

Trayendo un breve curriculum vitae del Prof. Dr. Claudio Rama, destacaremos su formación académica, su trayectoria académico-profesional y su producción bibliográfica. Nuestro entrevistado se graduó en Economía por la Universidad Central de Venezuela en 1982. En 2003 concluyó el máster en Administración Educacional en la Universidad José María Vargas, Venezuela. Terminó el Doctorado en Ciencias de la Educación en la Universidad Nacional Experimental Simón Rodríguez, Venezuela en 2006 y el Doctorado en Derecho, en la Universidad de Buenos Aires, en 2009. Claudio Rama hizo pos-doctorado: en el Centro de Postgrados de la Universidad Nacional Experimental Simón Rodríguez, en 2009-2010; en el Programa de Posgrado de la Faculdade de Educação da UNICAMP, en 2009-2010; en el Programa de Posgrado de la Universidade Federal Fluminense, en 2011-2012; y en el

\footnotetext{
1 Doctorado em Ciencias de la Educación en la Universidad Nacional Experimental Simón Rodríguez (Venezuela). Pos-doctorado em Derecho en la Universidad de Buenos Aires (Argentina). Centro de Educación Superior y Sociedad del Conocimiento / Facultad de Ciencias de la Educación / Universidad de la Empresa (Uruguay). E-mail: claudiorama@gmail.com. ORCID: http://orcid.org/0000-0002-4716-6572.

${ }^{2}$ Doctorado en Filosofía, Historia y Educación por la Universidad Estadual de Campinas (UNICAMP). Posdoctorado por la Universidad del São Paulo (USP) y UNICAMP. Docente investigadora del PPGEdu de la Unoesc. Joaçaba - SC / Brasil. E-mail: malu04@ gmail.com - ORCID: http://orcid.org/0000-0001-8515-2908.

${ }^{3} \mathrm{PhD}$ en Educación por la University of Southern California (USC). Pos-doctorado por la University of California at Los Angeles (UCLA). Profesor titular de la UNICAMP. Editor de la Revista RIESup de la FE de la UNICAMP. Campinas - SP / Brasil. E-mail: jcamilosantos@ gmail.com. ORCID: http://orcid.org/0000-00024183-0460.
}

v. 2

n. 1

p.214-219

jan./abr. 2016

ISSN 2446-9424 
Programa de Postdoctorado de la Facultad de Derecho, de la Universidad de Buenos Aires, en 2013-2015. Nuestro entrevistado recibió también el título de Doctor "honoris causa" de cuatro universidades peruanas.

En cuanto a su trayectoria académico-profesional, mencionaremos tan sólo las más relevantes. Trasterminar el curso de grado, Claudio Rama actuó como profesor de la Universidad Central de Venezuela, en el periodo de 1982 a 1985. De 1986 a 1994 fue profesor de la Universidad de la República del Uruguay y de 1994 a 2000, profesor de la Universidad de la Empresa del Uruguay (UDE). En 2001, fue contratado como director del Instituto Internacional para la Educación Superior y el Caribe (IESALC), de la UNESCO, con sede en Caracas (Venezuela), ahí actuando hasta 2006. En 2009 ejerció la función de investigador en la Facultad de Educación da Universidad de la Empresa (UDE), en 2010 se tornó el Decano de la Facultad de Ciencias Empresariales y en 2015, Director del Centro de Estudios de Educación Superior y Sociedad del Conocimiento (CEESSC), de la Facultad de Educación, de la UDE. Actualmente, es Rector del Instituto de Altos Estudios Nacionales (IAEN), la nombrada Universidad de Postgrado del Estado de Ecuador.

Claudio Rama publicó 25 libros y fue coeditor de 19 libros. Varios de ellos tratan de problemas de las universidades latinoamericanas, de las universidades del espacio del Mercosur y de las tendencias de la educación superior en el mundo contemporáneo de la sociedad de la información y del conocimiento, de la globalización y de la internet.

En este número de la Revista entrevistamos al Prof. Dr. Claudio Rama sobre dos temas básicos muy relevantes para la educación superior brasileña y latinoamericana: a) El Mercosur y la educación superior; e b) Tendencias de la educación superior. Sobre el primer tema, fueron hechas dos preguntas y sobre el segundo, cinco. En las respuestas a las preguntas, Claudio Rama sintetiza su punto de vista. Un análisis más profundizado de su visión sobre esos temas puede ser buscado en su vasta producción bibliográfica.

\section{Mercosur y educación superior}

\section{María de Lourdes y José Camilo - ¿Ve posibilidad de busca de simetría y consecuente reconocimiento automático de los diplomas de cursos de grado y de pos-grado de las universidades de los países del Mercosul en un futuro próximo?}

El reconocimiento automático de los títulos de graduación se basa en la existencia de dinámicas similares de los procesos académicos y niveles de calidad similares. La lógica del reconocimiento automático sólo puede existir si esas condiciones son iguales. Si las situaciones son diferenciadas hay una asimetría de situaciones de las personas ante los mercados de trabajo, en tanto se benefician de iguales oportunidades laborales pero sobre la base de diferencias de las competencias y capacidades. En el marco del ejercicio profesional, el reconocimiento automático de las certificaciones de los países del acuerdo de integración, dada la ausencia de iguales condiciones de producción y de calidad entre los

\begin{tabular}{l|l|l|l|l|l|l} 
(C) Rev. Inter. Educ. Sup. & Campinas, SP & v.2 & n.1 & p.214-219 & jan./abr. 2016 & ISSN 2446-9424
\end{tabular}


países, permite la aparición del dilema del polizón (problema free rider) por el cual una persona puede competir y acceder a derechos sin tener los mismos costos de los otros. Ello se realiza por la vía del "atajo educativo" por el cual, accede a una certificación producida en el exterior de un país y al interior del bloque que tiene el mismo valor que las otras certificaciones pero sobre la base de un nivel de capital humano inferior resultado de inferiores estándares y exigencias. Las certificaciones tienen igual valor de mercado, pero diferentes costos y exigencia y por ende derivan en diferentes niveles de formación y de capital humano.

El MERCOSUR Educativo en el marco del objetivo de buscar promover una movilidad y con miras a facilitar el reconocimiento automático de las certificaciones, estableció como requisito de los estudios de graduación y posgraduación una similar cantidad de horas. Sin embargo a pesar de haber sido ratificado por los Congresos Nacionales de los países, dada la autonomía, para el caso de Uruguay, de la UDELAR, o las normas de regulación del funcionamiento de las ofertas privadas, esas horas mínimas establecidas en el Protocolo del MERCOSUR no se cumplen en forma homogénea por los distintos países. Sin embargo, más allá de las horas mínimas de clase, existen enormes niveles de diferencia de los programas y de los sistemas de educación superior en América Latina y específicamente en el MERCOSUR que imposibilitan un reconocimiento automático de las titulaciones. Al interior del bloque, tanto a nivel de grado o de posgrado, hay múltiples diferencias de exigencias y de regulación de los docentes, de las formas de ingreso y egreso, del peso de las prácticas profesionales, de las exigencias curriculares, de las regulaciones, de las cargas docentes, de las tesis, etc., que hace prácticamente imposible pensar en que los procesos de reconocimiento automático de las certificaciones, no estén permitiendo el acceso de "polizones" o de "atajos" educativos, que reafirmen la inequidad y desigualdad de las personas.

\section{María de Lourdes y José Camilo - ¿En el rumbo de esta (¿posible?) simetría de cursos y diplomas equiparables, ve contribución de la experiencia de la Unión Europea con su proceso de Bolonia?}

El Acuerdo de Bolonia, constituye una experiencia muy coherente y a la vez una referencia de las características de la integración universitaria. Sin embargo, parte de una realidad altamente diferenciada entre Europa y América y más específicamente de los países del Mercosur. La Europa tradicional tiene un peso destacado de sistemas de educación públicos y donde relativamente había estándares relativamente comunes previos al Proceso de Bolonia. La región a diferencia tiene una alta diferenciación en el peso de la educación privada, en las estructuras institucionales, en los niveles de aseguramiento de la calidad o en las formas de ingreso y egreso, que limitan fuertemente la conformación de procesos de integración. A 16 años de inicio del proceso de Bolonia ha habido fuertes avances en Europa, mientras que a 31 años de inicio del Mercosur, los avances reales son muy reducidos. Sólo en el caso del posgrado, mientras que Brasil ha diferenciado el sistema entre latu y estricto senso, en los demás países, las especializaciones son posgrados regulados y

\begin{tabular}{l|l|l|l|l|l|l} 
(C) Rev. Inter. Educ. Sup. & Campinas, SP & v.2 & n.1 & p.214-219 & jan./abr. 2016 & ISSN 2446-9424 \\
\hline
\end{tabular}


tienen más importancia frente a las maestrías y doctorados. Los sistemas de remuneración y de exigencias de calidad, claramente diferencian la importancia de las certificaciones de maestría y doctorado entre los países. No veo posible en el corto ni mediano plazo, que los sistemas de educación superior de la región converjan a través de un proceso de integración educativo al estilo del Proceso de Bolonia. El grado de autonomía de las universidades, los diferentes marcos de políticas nacionales y las distintas concepciones sobre la dinámica universitaria, torna muy difícil visualizar un proceso como ese, más allá de que finalmente los procesos de integración universitarios se apoyan en procesos de integración económicos y sociales profundos y no meras uniones aduanales incompletas que es lo más dominantes en la subregión del Mercosur.

\section{Tendencias de la educación superior}

\section{María de Lourdes y José Camilo - ¿En qué la tendencia para la sociedad de la información y del conocimiento afectará a la estructura y a la organización de la universidad del futuro próximo?}

La lenta construcción de sociedades de información o de conocimiento ya está afectando fuertemente las estructuras o formas de organización de las universidades. Los componentes más característicos de ello son el aumento del peso de los posgrados, la mayor orientación hacia la investigación, el creciente peso de procesos de internacionalización de las dinámicas educativas. Los posgrados expresan la expansión del conocimiento y la investigación en la base de la nueva sociedad del capitalismo cognitivo. El aumento de dinámicas de internacionalización de estudiantes, docentes, currículos e instituciones, constituye una expresión de estos cambios societarios que están recomponiendo el funcionamiento de las universidades. En este contexto, es de destacar también la creciente diferenciación de modalidades y de ofertas como expresión del aumento de la división social y técnica del trabajo que caracteriza a la nueva sociedad en construcción. Dentro de ello, es de destacar la virtualización y la universidad en red como componente asociado a lo internacional, lo tecnológico y lo pedagógico. Se sostiene que en general, la mayor parte de las mutaciones universitarias finalmente están asociadas a la lenta conformación de sociedades de la información y del conocimiento que caracteriza la actual fase del capitalismo cognitivo que expande la educación superior.

\section{María de Lourdes y José Camilo - ¿En qué la consolidación de internet en el mundo afectará la estructura y la organización de la universidad del futuro próximo?}

La virtualización y la sociedad en redes digitales están transformando radicalmente la dinámica del acceso, la creación, la distribución y el uso del conocimiento. Internet, como expresión de nuevas relaciones sociales, se constituye en el basamento de una sociedad global y donde la educación, la cultura y la investigación se digitalizan y promueven su acceso a través de redes digitales. La universidad de papel, presencial, nacional, de tiza, lengua y pizarrón, está siendo suplantada y reconfigurada con las redes digitales que

\begin{tabular}{l|l|l|l|l|l|l} 
(C) Rev. Inter. Educ. Sup. & Campinas, SP & v.2 & n.1 & p.214-219 & jan./abr. 2016 & ISSN 2446-9424
\end{tabular}


promueven nuevas pedagogías informáticas, la educación virtual, los recursos de aprendizaje digitales entre los cuales destacadamente los software y sistemas de simulación como ejes de un aprendizaje autónomo. La educación automática que promoviera Skinner en los años 50 está expresándose hoy en los MOOCs (Massive Open Online Couses) que ponen en el escenario nuevas globouniversidades, un cambio del trabajo docente con un mayor peso en el trabajo muerto, y un rol destacado en sistemas de aprendizaje automatizado y planificados previamente. La educación del futuro no será presencial ni a distancia sino digital, imponiendo cambios enormes en el rol del auto aprendizaje programado. Estamos en una fase de innovación educativa enorme, sobre la base del desarrollo de nuevas tecnologías de comunicación e información (holograma, redes semánticas, inteligencia artificial, máquinas de enseñanza) que cambiarán con mayor intensidad los viejos paradigmas educativos y las formas de la enseñanza y el aprendizaje.

María de Lourdes y José Camilo - ¿En qué aspectos la educación superior a distancia complementará e incluso sustituirá la educación superior presencial?

El debate de Frankfurt y las transformaciones en la cultura con la irrupción de las industrias culturales, constituyó una referencia que para algunos en algún momento nos permitió visualizar una educación de masas estandarizada barata y una educación presencial personalizada cara, sobre la base de la contrastación con la televisión y la radio, frente a la ópera, el teatro o el ballet. Sin embargo, los escenarios son muchos más complejos en los entornos digitales actuales que en los entornos analógicos donde se conformaron las industrias culturales hertzianas.

Estamos viendo un lento y persistente avance de la educación a distancia o virtual frente a la educación presencial. He sostenido que en el actual contexto las barreras entre ambas modalidades están desapareciendo e irrumpe una educación digital que puede ser tanto presencial como a distancia. Actualmente vemos algunos procesos de complementación con plataformas digitales, clases invertidas, software y recursos de auto aprendizaje, pero la tendencia en algunas áreas puede ser de una sustitución muy elevada. Es difícil hacer prospectiva en esta materia, y en el área de ciencia y tecnologías, desde los estudios de Herman Khan en los 70 se ha verificado la falacia de la futurología en la materia. Estamos recién en los inicios de la autopista de la información y ello hace más compleja la prospectiva, más allá de que esta no es una ciencia.

María de Lourdes y José Camilo - ¿En qué aspectos la educación superior presencial y la vivencia universitaria continuarán siendo esenciales para una educación superior de excelencia?

Hace algunos años se sostenía, más en forma ideológica que empírica, que la calidad era la presencialidad. La no presencialidad era un indicador de baja calidad en los aprendizajes. Hoy la evaluación de los estudiantes de distintas modalidades muestra que las diferencias no son significativas. Más aún se verifica que en algunos casos, con poblaciones similares, los

\begin{tabular}{l|l|l|l|l|l|l} 
(C) Rev. Inter. Educ. Sup. & Campinas, SP & v.2 & n.1 & p.214-219 & jan./abr. 2016 & ISSN 2446-9424
\end{tabular}


aprendizajes son superiores. La imagen y el sonido superan al docente catedrático, la práctica a la teoría, y la simulación y el acceso a información global, a la enseñanza en el aula. Sin embargo, es también claro que la conformación de ciudadanos, de redes sociales, de reales intercambios cognitivos, de aprendizajes prácticos o de construcción de valores, requiere una actividad presencial y la vivencia. Como sostenía Jasper, no tendríamos vivencia real, que es uno de los componentes destacados del aprendizaje, sin la presencia física de las personas.

\section{María de Lourdes y José Camilo - ¿Cuál es el futuro de la interdisciplinaridad en la universidad?}

La educación del futuro, y especialmente en los niveles inferiores del grado, tenderá a ser cada vez más interdisciplinaria. El modo 2 de creación del conocimiento muestra la eficacia cognitiva en la superación del paradigma de Descartes y Kan del modo 1 de creación del conocimiento por la vía de la fragmentación disciplinaria. La complejidad del conocimiento y su carácter sistémico, tornan central los enfoques interdisciplinarios. También las profesiones están cambiando y el tipo de trabajo de los universitarios está cambiando. La irrupción de universidades, currículos, enfoques y análisis interdisciplinarios, se constituye en uno de los ejes futuros. El avance hacia la investigación y la focalización en problemas, muestra la superación de las estructuras disciplinarias y de las organizaciones en campos disciplinarios. Sin duda aparecerán nuevos paradigmas gracias a los avances de la neurociencia y la propia investigación aplicada a escala global. El trabajo en red y el uso de sistemas informáticos aportarán nuevos avances. Todos ellos apuntan hacia la dinámica colectiva en la construcción de conocimientos, y en tal sentido hacia el avance hacia el conocimiento en red y a la propia universidad en red. Castells ya apuntaba a una sociedad de información soportada en redes digitales, con e-negocios e instituciones en red que permite formular la creciente irrupción de universidades en red- sean o no virtualesaprendizaje y e investigación en red y también formular modelos multi-interdisciplinarios en el marco de redes sociales, académicos, económicas e institucionales como paradigmas epistemológicos futuros. 